\title{
Caracterización trófica del placóforo intermareal Enoplochiton niger en el norte de Chile: variación ambiental y patrones dietarios a nivel local y regional
}

\author{
Trophic characterization of the intertidal placophoran Enoplochiton niger in northern \\ Chile: environmental variation and dietary patterns at local and regional levels
}

ÁlVARO G. SANHUEZA ${ }^{1}$, ARTURO H. NAVARRETE ${ }^{1}$, L. FELIPE OPAZO ${ }^{1}$ \& PATRICIO A. CAMUS P $^{1,2}$

\author{
${ }^{1}$ Departamento de Ecología Costera, Facultad de Ciencias, \\ Universidad Católica de la Santísima Concepción, Casilla 207, Concepción, Chile \\ ${ }^{2}$ Center for Advanced Studies in Ecology \& Biodiversity (CASEB) \\ * e-mail para correspondencia: pcamus@ucsc.cl
}

\begin{abstract}
RESUMEN
El rol e impacto de los herbívoros en la trama trófica de comunidades intermareales rocosas podría ser más diverso y complejo de lo considerado hasta ahora, particularmente en el caso de consumidores de mayor tamaño y abundancia como el molusco placóforo Enoplochiton niger. Esta especie es uno de los pastoreadores de mayor tamaño (hasta $20 \mathrm{~cm}$ ) e importancia ecológica en las costas rocosas del norte de Chile, pero también una de las especies menos conocidas en términos tróficos. Este trabajo presenta una evaluación de los patrones dietarios de E. niger en cuatro comunidades del norte de Chile, distribuidas en $1.000 \mathrm{~km}$ de costa y muestreadas estacionalmente entre invierno 2004 y otoño 2006. Además se analizó la relación de su dieta con factores biológicos y físicos, incluyendo el efecto potencial del evento El Niño 2004-2005 ocurrido durante el período de estudio. A nivel regional, el espectro dietario de E. niger abarcó un total de 98 recursos (60 ítemes algales y 38 ítemes invertebrados), y los ítemes más importantes fueron organismos sésiles con formas de crecimiento incrustante o en capa. E. niger mostró una gran amplitud de nicho tanto a nivel regional como local (rango: 20,7-28,0; índice de Levins), con una riqueza dietaria a nivel individual independiente del tamaño corporal. Tanto el número de ítemes dietarios consumidos por individuo como la composición taxonómica de la dieta no mostraron diferencias significativas entre comunidades, pero variaron significativamente entre el período asociado al evento El Niño y el período posterior. No hubo ninguna relación clara entre los patrones dietarios de E. niger y los niveles contrastantes de intensidad de surgencia entre las comunidades estudiadas. Los resultados muestran que E. niger es un consumidor generalista y polífago, y un potencial omnívoro, el cual podría tener un alto impacto sobre los patrones de ocupación de espacio en la comunidad intermareal.
\end{abstract}

Palabras clave: chitón, tamaño corporal, omnivoría, surgencia, El Niño.

\begin{abstract}
The role and impact of herbivores on rocky intertidal food webs could be more complex and diverse than previously considered, particularly in the case of larger and more abundant consumers such as the placophoran mollusc Enoplochiton niger. This species is one of the largest (up to $20 \mathrm{~cm}$ ) and ecologically most important grazers on rocky shores of northern Chile, but also one of the lesser known species in trophic terms. This work presents an assessment of the dietary patterns of E. niger in four communities of northern Chile, distributed along $1,000 \mathrm{~km}$ of coastline and sampled seasonally from winter 2004 to autumn 2006 . The analysis included the relationship of its diet with physical and biological factors, and particularly the potential effect of the 2004-2005 El Niño event that occurred during the study period. At a regional level, the dietary spectrum of E. niger comprised a total of 98 food resources (60 algal items and 38 invertebrate items), and the most important items were sessile organisms of encrusting and layer-forming growth habits. E. niger exhibited a very high niche breadth at both local and regional levels (ranging from 20.7 to 28.0; Levins' index), and its dietary richness at the individual level was independent from its body size. Both the number of dietary items consumed per individual and the taxonomic composition of the diet did not show statistical differences among communities, but they were significantly different between the El Niño and non-El Niño periods. There was no clear relationship between the dietary patterns of E. niger and the contrasting levels of
\end{abstract}


upwelling intensity among the communities studied. The results show that E. niger is a generalist polyphagous consumer, and a potential omnivore, which could have a high level of impact on space occupancy patterns in the intertidal community.

Key words: chiton, body size, omnivory, upwelling, El Niño.

\section{INTRODUCCIÓN}

Los placóforos o chitones son un grupo tróficamente diverso que incluye mayormente especies herbívoras pero también carnívoras, omnívoras, detritívoras, epizóofagas y xilófagas (e.g., Latyshev et al. 2004). A nivel mundial son reconocidos por su particular importancia en sistemas intermareales rocosos, y su capacidad de generar una fuerte presión de pastoreo afectando la estructura comunitaria (Boyle 1977, Lubchenco \& Gaines 1981, Hawkins \& Hartnoll 1983, Otway 1994, Markel \& De Wreede 1998). Por ello las especies más estudiadas han sido aquellas de hábitos principalmente herbívoros, que tienden a consumir una alta proporción de microalgas y algas filamentosas pequeñas (Boyle 1977, Steneck \& Watling 1982, Hawkins \& Hartnoll 1983, Otaíza \& Santelices 1985, Otaíza 1986, Santelices et al. 1986). Sin embargo, diversos antecedentes muestran que la conducta y hábitos tróficos de los chitones pueden variar por ejemplo en función de sus patrones de uso del hábitat y de las variaciones locales, geográficas y estacionales en el tipo y número de recursos disponibles (e.g., Lubchenco \& Gaines 1981, Langer 1983, Santelices et al. 1986, Smith \& Otway 1997, Kelaher \& Cole 2005). Algunas especies incluso pueden alternar entre una dieta herbívora y una omnívora dependiendo del ambiente en que se encuentren (e.g., Latyshev et al. 2004), aunque el consumo de ítemes animales parece ser un fenómeno común en este grupo (e.g., Camus et al. 2008).

En la costa del Pacífico sudamericano, una de las especies más conspicuas de chitones es Enoplochiton niger (Barnes, 1824), el cual se distribuye latitudinalmente entre $\operatorname{los} 15^{\circ}$ y $30^{\circ} \mathrm{S}$ (Valdovinos 1999). En el norte de Chile, E. niger es uno de los herbívoros más comunes y abundantes en las zonas intermareales rocosas medias y bajas, y además particularmente importante por su gran tamaño que puede superar los $15 \mathrm{~cm}$ en longitud (Camus 1994). En esta región, E. niger, junto a otras especies como
Acanthopleura echinata (Barnes, 1824) y Chiton granosus (Frembly, 1827), y secundariamente Tonicia spp., son consumidores de alta relevancia en las comunidades intermareales debido a su gran amplitud trófica (Camus 1994, Camus et al. 2008). Recientemente, Camus et al. (2008) han mostrado que todas las especies de chitones del norte de Chile son omnívoros tróficos (sensu Pimm \& Lawton 1978) y potencialmente omnívoros fisiológicos, si bien aún no se ha evaluado concluyentemente su capacidad de digerir y asimilar alimento animal (aunque algunas evidencias preliminares en $C$. granosus indican que al menos esta especie efectivamente lo hace; Cid 2007¹). Por ello, un conocimiento más preciso de la dieta de los chitones resulta esencial para clarificar su grado de conectividad y posición trófica en los flujos de energía de la trama intermareal.

Sin embargo, en Chile aún no se ha evaluado si la alimentación de estos chitones podría ser afectada por las fluctuaciones físicas y biológicas que modulan tanto la calidad y disponibilidad de sus recursos como la productividad primaria. Entre estos procesos sobresalen las variaciones de mesoescala en la intensidad de surgencia y suministro de nutrientes, que inciden diferencialmente en el crecimiento algal y pueden determinar el impacto de un herbívoro en la comunidad (Nielsen \& Navarrete 2004), y también la variación interanual ligada a los eventos El Niño y La Niña, capaz a su vez de modificar la intensidad y efectos de la surgencia (véase revisión de Thiel et al. 2007). Ambos factores son de particular relevancia en la costa norte de Chile, donde sus efectos interactúan de forma compleja afectando a las poblaciones de herbívoros y sus patrones de consumo de recursos (e.g., Vega et al. 2005, Thiel et al. 2007, Camus 2008).

1 CID Y (2007) Evaluación de la asimilación de alimento animal en Chiton granosus (Frembly, 1827) y su estrategia trófica como potencial omnívoro. Seminario de investigación, Facultad de Ciencias, Universidad Católica de la Santísima Concepción, Concepción, Chile. 13 pp. 
En este contexto, presentamos una evaluación de la riqueza y composición dietaria de Enoplochiton niger en el norte de Chile, evaluadas estacionalmente durante dos años en cuatro comunidades intermareales rocosas distribuidas en $1.000 \mathrm{~km}$ de costa. Los resultados incluyen el análisis de los patrones de variación espacial y temporal en la dieta de E. niger, y su relación con factores biológicos como el tamaño corporal y factores físicos como la temperatura superficial del mar. En particular discutimos los posibles efectos del evento El Niño 2004-2005 ocurrido en el período abarcado por este trabajo (CPC 2007).

\section{MATERIALES Y MÉTODOS}

\section{Obtención de datos}

El estudio se llevó a cabo en cuatro comunidades intermareales rocosas del norte de Chile abarcando ca. de $10^{\circ}$ de latitud (Fig. 1), las cuales presentan niveles contrastantes en intensidad de surgencia (Vásquez et al. 1998, Camus \& Andrade 1999, Thiel et al. 2007): Río Seco $\left(21^{\circ} 00{ }^{\prime} 05^{\prime}\right.$ S, $70^{\circ} 09^{\prime} 54 " \mathrm{O}$; sur de Iquique, baja surgencia), Caleta Constitución $\left(23^{\circ} 25^{\prime} 23 "\right.$ S, $70^{\circ} 35^{\prime} 26^{\prime \prime} \mathrm{O}$; norte de Antofagasta, alta surgencia), Caleta Angosta (2815'38' S, $71^{\circ} 10^{\prime} 23^{\prime \prime}$ O; norte de Huasco, baja surgencia), y Lagunillas $\left(30^{\circ} 06^{\prime} 14^{\prime \prime} \mathrm{S}\right.$, $71^{\circ} 22^{\prime} 57^{\prime \prime}$ O; sur de Coquimbo, alta surgencia). Las cuatro comunidades fueron muestreadas estacionalmente en: invierno 2004 (15-30 agosto), primavera 2004 (8-15 noviembre), verano 2005 (20-26 enero), otoño 2005 (22-28 mayo), invierno 2005 (18-24 agosto), primavera 2005 (8-15 noviembre), verano 2006 (27 enero-5 febrero) y otoño 2006 (21-29 mayo).

En cada muestreo se evaluó para cada comunidad la presencia y abundancia de especies sésiles y móviles en forma no destructiva, en un total de 45 cuadrantes $(0,25$ $\mathrm{m}^{-2}$ ) distribuidos en cinco bloques (posicionados mediante GPS) espaciados ca. 15 $\mathrm{m}$ entre sí. Cada bloque incluyó tres series de cuadrantes dispuestos en las zonas intermareales alta, media y baja (tres cuadrantes por zona) espaciados entre sí ca. 1,5 y $5 \mathrm{~m}$ en sentido vertical y horizontal respectivamente. En cada muestreo se registró la temperatura superficial del mar (en adelante TSM) mediante mediciones manuales con un termómetro, y desde "data loggers" (Onset Stowaway) instalados $1 \mathrm{~m}$ bajo el nivel medio de marea baja. Con objeto de evaluar la dieta de $E$. niger e incorporar la variación espacial sus recursos tróficos en cada comunidad, desde cada localidad se colectaron entre seis y 10 individuos por muestreo desde las zonas intermareales media y baja y en los cinco bloques de muestreo. Cada individuo colectado fue inyectado y almacenado con formaldehído $10 \%$ para preservar de buena forma su contenido gástrico, y facilitar así la identificación de los ítemes dietarios. En el laboratorio, cada individuo fue medido (longitud máxima) y pesado (biomasa seca), extrayendo su contenido gástrico mediante disección, el cual fue analizado bajo microscopio y lupa estereoscópica, identificando cada ítem dietario al nivel taxonómico más bajo posible.

\section{Análisis}

Los análisis descritos a continuación se basan en datos de dieta obtenidos en terreno, que pueden evaluarse a múltiples escalas y que en algunos casos no tienen una correspondencia uno a uno. En función de las variables a evaluar, se han seleccionado combinaciones de datos y niveles de análisis abarcando aspectos regionales y locales para proveer una adecuada caracterización dietaria. En los análisis de varianza utilizados se evaluó previamente el cumplimiento de los supuestos necesarios (homocedasticidad y normalidad) mediante distintas pruebas, mencionando aquellos casos en que debió efectuarse una transformación correctiva de los datos. Las comunidades de estudio fueron elegidas por sus características contrastantes y para abarcar las zonas representativas del norte de Chile, por lo cual se consideraron un efecto fijo en ANDEVA, mientras que las estaciones muestreadas fueron definidas como efecto aleatorio. La variación en biomasa de los individuos de E. niger colectados en las cuatro comunidades y ocho estaciones fueron evaluadas mediante ANDEVA de dos vías, usando una prueba de Tukey para comparación a posteriori. Adicionalmente se estimó la relación entre biomasa seca y longitud máxima mediante un 
ajuste de regresión. Para comparar la densidad de E. niger entre comunidades se efectuó una prueba de Kruskal-Wallis debido a la falta de normalidad y homocedasticidad de los datos, aplicando una prueba a posteriori para comparación de medias obtenidas por ranking (Siegel \& Castellan 1988).

\section{Dieta}

A partir del análisis de contenidos gástricos de E. niger se obtuvo el número de ítemes dietarios (riqueza dietaria en adelante) consumido por cada individuo analizado, a partir del cual se calculó la frecuencia de ocurrencia de cada ítem en el conjunto de individuos muestreados por comunidad. En base a valores promedio calculados por estación y comunidad, se evaluó la relación entre la biomasa de los individuos de E. niger y su riqueza dietaria mediante análisis de correlación de Spearman, considerando tanto la riqueza dietaria total de ítemes como la de ítemes algales y animales por separado.

La variación de la riqueza dietaria por individuo entre comunidades y estaciones se evaluó mediante ANDEVA de dos vías, con datos transformados a $\log _{10}(x+1)$, aplicando una prueba de Tukey para comparación de medias. La relación regional entre la TSM y la riqueza dietaria se evaluó mediante correlación de Spearman usando los valores de riqueza dietaria promedio por estación. Para cada grupo de individuos de E. niger colectados en cada estación y comunidad, se estimó su amplitud de nicho trófico mediante el índice de Levins (recíproco del índice de Simpson; Levins 1968) usando la riqueza y frecuencia de ocurrencia de los ítemes dietarios registrados. La variación en amplitud de nicho entre comunidades se analizó mediante ANDEVA de una vía, en base a los valores respectivos de amplitud de nicho por estación.

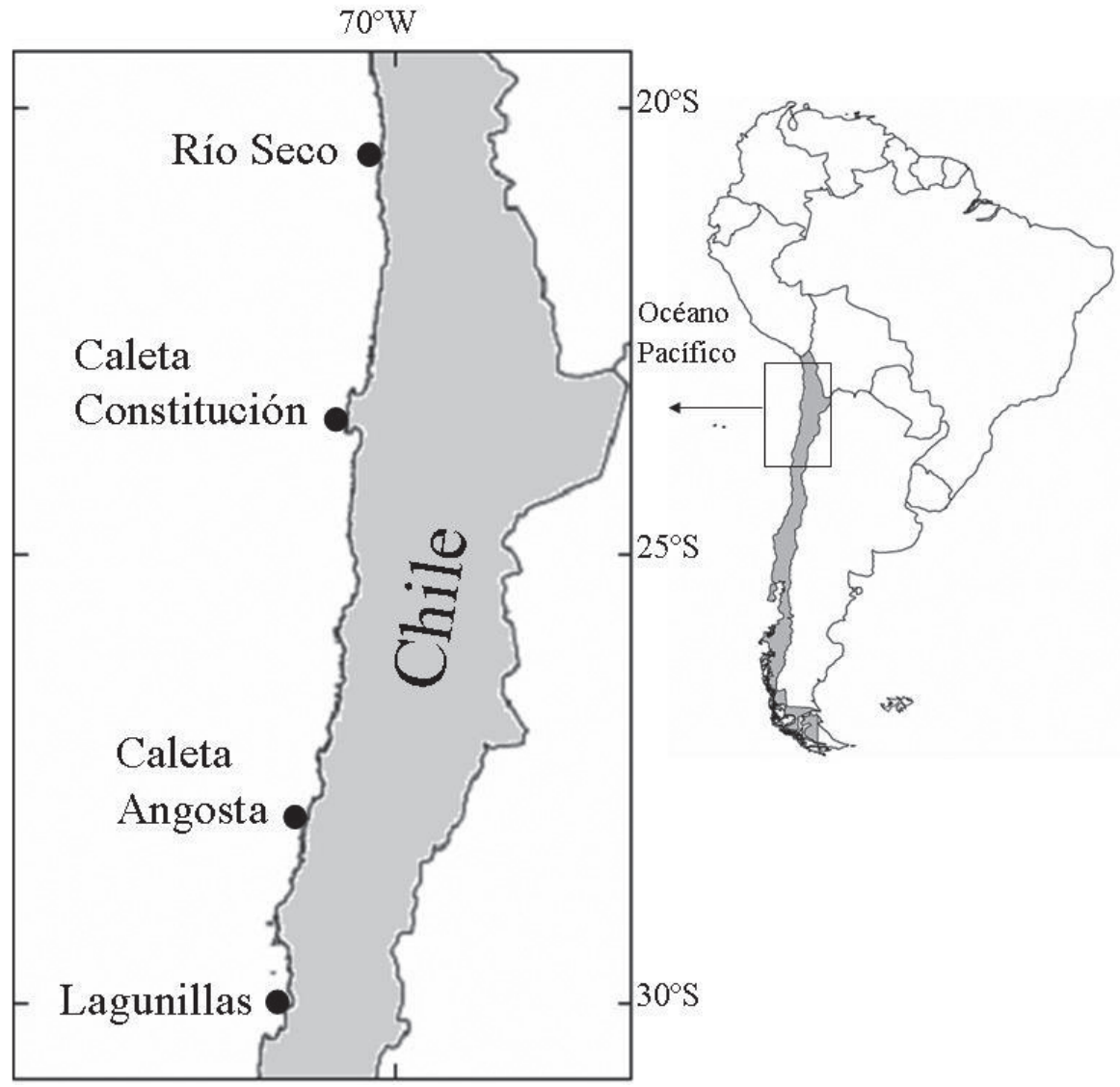

Fig. 1: Localización de las comunidades de estudio en el norte de Chile.

Location of study communities in northern Chile. 
Por último, la variación espacial y temporal en la composición de la dieta de E. niger se evaluó mediante un análisis de escalamiento multidimensional no métrico (nMDS), que se basó en una matriz de similitud de Bray-Curtis calculada desde los valores de frecuencia de ocurrencia de los ítemes dietarios en cada combinación de comunidad*estación de muestreo. Posteriormente se realizó un análisis de similitud (ANOSIM, Clarke 1993) para evaluar diferencias entre comunidades y/o de estaciones. En este análisis se incorporó el factor presencia versus ausencia del evento El Niño 2004-2005 durante el período de estudio, para evaluar el posible efecto de este evento en la composición trófica de E. niger a nivel regional.

\section{RESULTADOS}

Distribución vertical, densidad y tamaño corporal

La distribución vertical de E. niger en el norte de Chile se restringió a los niveles intermareales medio y bajo, donde se encontró respectivamente al 25,9 y $74,1 \%$ de 1 los individuos, en 116 de los 960 cuadrantes muestreados en ambas zonas (i.e., 12,1\% de ocurrencia regional). La Fig. 2 muestra la densidad de E. niger por nivel mareal en cada comunidad, evidenciando su mayor ocurrencia en la zona baja en todos los casos, una diferencia que fue particularmente notoria en las dos localidades de mayor latitud que abarcan el extremo sur de su distribución geográfica. La densidad de E. niger a nivel regional y comunitario (Tabla 1) fue relativamente baja (en parte porque su cálculo incluyó los cuadrantes con valor cero), y mostró diferencias significativas entre comunidades $(\mathrm{H}=26,53 ; \mathrm{gl}=3 ; \mathrm{n}=958 ; \mathrm{P}<$ $0,0001)$ asociadas a una disminución gradual con la latitud. No obstante, la prueba a posteriori no detectó diferencias significativas entre pares de comunidades, evidenciando que la densidad de E. niger no decae drásticamente hacia el límite de su distribución.

El tamaño corporal promedio de los individuos de E. niger colectados en terreno fue de 29,20 $\pm 1,11$ (EE) g en biomasa seca, y de $12,18 \pm 0,21(\mathrm{EE}) \mathrm{cm}$ en longitud máxima. Entre estos individuos, aquellos de mayor tamaño alcanzaron una biomasa seca de 80,85 g y una longitud de 19,30 cm. En particular la biomasa seca por individuo (Tabla 1) fue significativamente distinta entre localidades

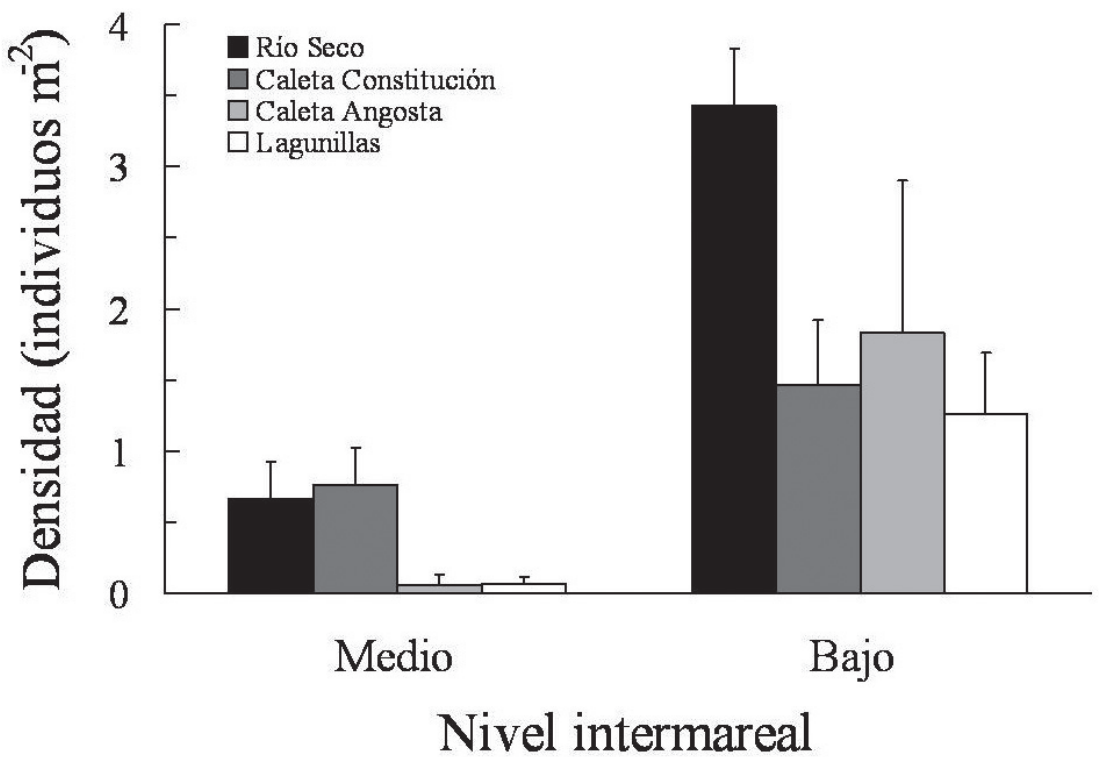

Fig. 2: Densidad de Enoplochiton niger en los niveles intermareales medio y bajo en las comunidades de estudio. Los valores corresponden a medias \pm EE. 
$\left(\mathrm{F}_{3,246}=15,29 ; \mathrm{P}<0,0001\right)$ y estaciones $\left(\mathrm{F}_{7,246}\right.$ $=3,57 ; \mathrm{P}=0,0109)$, con una interacción significativa entre ambos factores $\left(\mathrm{F}_{21,246}=\right.$ 2,83; $\mathrm{P}<0,0001)$. La interacción se debió a una fluctuación estacional concomitante entre Río Seco y Caleta Constitución (localidades más al norte), donde la biomasa promedio no solo aumentó hacia el verano y disminuyó hacia el invierno, sino que además fue menor (prueba de Tukey; $\mathrm{P}<0,05$ en todas las comparaciones) que en Caleta Angosta y Lagunillas (localidades más al sur) donde fluctuó sin tendencia definida. Esta variación en biomasa individual en las cuatro comunidades no mostró ninguna relación aparente con la ocurrencia del evento El Niño 2004-2005. Por otra parte, la biomasa (B) de E. niger tuvo una buena correspondencia con su longitud (L), relacionándose por la función $\mathrm{B}=0,0303 \mathrm{~L}^{2,650}$ $\left(\mathrm{R}^{2}=0,850 ; \mathrm{n}=278 ; \mathrm{P}<0,0001\right)$. Los residuos de esta relación fueron homogéneos pero de magnitud notoriamente menor en los individuos más pequeños, evidenciando una mayor irregularidad en las proporciones corporales de los individuos mayores. Por otra parte, el tamaño corporal (biomasa) de E. niger no mostró relaciones significativas con el número de ítemes animales $\left(\mathrm{r}_{\mathrm{s}}=-0,250 ; \mathrm{n}=32 ; \mathrm{P}=\right.$ $0,166)$ o de ítemes algales $\left(\mathrm{r}_{\mathrm{s}}=-0,220 ; \mathrm{n}=32\right.$; $\mathrm{P}=0,226)$ consumidos en la dieta.

\section{Riqueza y amplitud dietaria}

El análisis de contenidos gástricos se basó en 278 individuos colectados en todas las comunidades y estaciones muestreadas. La dieta de E. niger estuvo compuesta por un total de 98 ítemes a nivel regional, distribuidos en $38(38,8 \%)$ ítemes invertebrados y $60(61,2 \%)$ ítemes algales. El grupo principal de ítemes algales estuvo compuesto a su vez por un 28,6 $\%$ de clorófitas, $21,4 \%$ de feófitas y $50 \%$ de rodófitas. La Tabla 2 presenta los ítemes dietarios registrados y su frecuencia de ocurrencia tanto a nivel regional como en cada comunidad estudiada. Los órdenes y miembros más representativos de los ítemes algales consumidos por E. niger fueron: (a) Chlorophyta: Caulerpales (Codium) y Ulvales (Ulva, Enteromorpha, Pseudoulvella); (b) Phaeophyta: Ectocarpales (Ectocarpus, Ralfsia); y (c) Rhodophyta: Ceramiales (Polysiphonia), Cryptonemiales (coralinas incrustantes en general), Gelidiales (Gelidium), Gigartinales (Gastroclonium, Hypnea, Schottera) e Hildenbrandiales (Hildenbrandia). Por otra parte, entre los ítemes animales, los cirripedios fueron claramente mayoritarios (70,5\% como ítem general y como especies), seguidos por briozoos y esponjas (Demospongiae).

TABLA 1

Atributos básicos de Enoplochiton niger en la zona intermareal rocosa del norte de Chile, evaluados a nivel comunitario y regional. La amplitud de nicho (índice de Levins) por comunidad fue calculada como promedio estacional y como valor global (estaciones agrupadas, entre paréntesis). Los valores corresponden a medias $\pm \mathrm{EE}$

Basic attributes of Enoplochiton niger in the rocky intertidal zone of northern Chile, assessed at community and regional levels: No. of sampled individuals, density, body size (dry biomass), and niche breadth (Levins' index) per community estimated as mean seasonal value and overall value (pooled seasons; in parenthesis). Values represent mean $\pm \mathrm{SE}$

\begin{tabular}{lccccc}
\hline Atributo & Río Seco & Caleta Constitución & Caleta Angosta & Lagunillas & Regional \\
\hline Número de individuos & 360 & 360 & 360 & 359 & 1.439 \\
Densidad (indiv m ${ }^{-2}$ ) & $2,03 \pm 0,37$ & $1,12 \pm 0,21$ & $0,95 \pm 0,30$ & $0,67 \pm 0,21$ & $1,19 \pm 0,14$ \\
$\begin{array}{l}\text { Tamaño corporal } \\
\text { (g biomasa seca) }\end{array}$ & $20,30 \pm 1,58$ & $20,84 \pm 2,14$ & $40,84 \pm 1,90$ & $36,61 \pm 2,18$ & $29,20 \pm 1,11$ \\
Amplitud de nicho & $16,4 \pm 2,59$ & $13,19 \pm 1,41$ & $13,73 \pm 1,70$ & $16,76 \pm 2,40$ & 28,04 \\
& $(27,82)$ & $(20,69)$ & $0(24,86)$ & $(28,54)$ & \\
\hline
\end{tabular}


TABLA 2

Ítemes dietarios consumidos por Enoplochiton niger en el norte de Chile, separados por grupo taxonómico y listados en orden decreciente según su frecuencia de ocurrencia regional. Columnas dos a seis: frecuencia de ocurrencia (\%) por comunidad y regional (REG). Columnas siete y ocho: ocurrencia temporal (proporción de estaciones muestreadas; \%) en los períodos de presencia (EN-P) y ausencia (EN-A) del evento El Niño 2004-2005. RS: Río Seco; CC: Caleta Constitución; CA: Caleta Angosta; LA: Lagunillas. nd: ítem no identificado a nivel taxonómico inferior

Dietary items consumed by Enoplochiton niger in northern Chile, separated by taxonomic group and listed in decreasing order following their regional occurrence frequency. Columns two to six: occurrence frequency (\%) per community (abbreviated names) and at regional (REG) level; Columns seven and eight: temporal occurrence (proportion of sampling seasons; \%) within the periods of presence (EN-P) and absence (EN-A) of the 2004-2005 El Niño event. RS: Río Seco, CC:

Caleta Constitución, CA: Caleta Angosta, LA: Lagunillas. nd: item not identified at lower taxonomic level

\begin{tabular}{lllllllll}
\hline Ítem trófico & RS & CC & CA & LA & REG & EN-P & EN-A \\
\hline
\end{tabular}

Rhodophyta

Corallinaceae incrustantes

Hildenbrandia lecanellieri Harriot

Gelidium chilense (Montagne) Santelices \& Montalva

Amphiroa dimorphan Lemoine

Schottera nicaeensis (Lamouroux ex Duby) Guiry \& Hollenberg

Polysiphonia sp.

Hypnea cenomyce J. Agardh

Amphiroa peruana Areschoug

Corallina officinalis (Decaisne) Kützing

Ceramium rubrum (Hudson) C. Agardh

Erytrotrichia carnea (Dillwin) J. Agardh

Polysiphonia pacifica Hollenberg

Porphyra columbina Montagne

Acrochaetium grandis (Levring) Papenfuss

Chondrus canaliculatus (C. Agardh) Greville

Montemaria horridula (Montagne) Joly \& Alveal

Hildenbrandia sp.

Gelidium lingulatum Kützing

Gastroclonium cylindricum Santelices, Abbott \& Ramírez

Polysiphonia mollis Hooker \& Harvey

Centroceras clavulatum (C. Agardh) Montagne

Polysiphonia paniculata Montagne

Chondria californica (Collins) Kylin

Pterosiphonia pennata (C. Agardh) Falkenberg

Rhodymenia skottsbergii (Dawson)

Gelidium pusillum (Stackhouse) Le Jolis

Gigartina tuberculosa (Harvey) Grunow

Bangia atropurpurea (Roth) C. Agardh

$\begin{array}{ccccccc}81,6 & 80,0 & 95,5 & 87,7 & 86,0 & 94,6 & 80,1 \\ 56,6 & 60,0 & 58,2 & 70,8 & 61,2 & 50,0 & 68,7 \\ 32,9 & 52,9 & 29,9 & 46,2 & 40,3 & 40,2 & 40,4 \\ 25,0 & 10,0 & 38,8 & 40,0 & 28,1 & 30,4 & 26,5 \\ 21,1 & 20,0 & 4,5 & 23,1 & 17,3 & 19,6 & 15,7 \\ 7,9 & 18,6 & 13,4 & 10,8 & 12,6 & 6,3 & 16,9 \\ 0 & 38,6 & 7,5 & 0 & 11,5 & 7,1 & 14,5 \\ 10,5 & 1,4 & 17,9 & 12,3 & 10,4 & 11,6 & 9,6 \\ 7,9 & 7,1 & 13,4 & 13,8 & 10,4 & 4,5 & 14,5 \\ 3,9 & 14,3 & 13,4 & 4,6 & 9,0 & 22,3 & 0 \\ 5,3 & 10,0 & 7,5 & 13,8 & 9,0 & 1,8 & 13,9 \\ 2,6 & 1,4 & 31,3 & 0 & 8,6 & 0 & 14,5 \\ 9,2 & 8,6 & 1,5 & 12,3 & 7,9 & 1,8 & 12,0 \\ 2,6 & 8,6 & 4,5 & 15,4 & 7,6 & 5,4 & 9,0 \\ 5,3 & 14,3 & 4,5 & 6,2 & 7,6 & 0 & 12,7 \\ 0 & 11,4 & 4,5 & 6,2 & 5,4 & 6,3 & 4,8 \\ 2,6 & 8,6 & 7,5 & 0 & 4,7 & 0 & 7,8 \\ 1,3 & 0 & 16,4 & 0 & 4,3 & 0 & 7,2 \\ 3,9 & 0 & 9,0 & 1,5 & 3,6 & 8,0 & 0,6 \\ 0 & 2,9 & 10,4 & 0 & 3,2 & 5,4 & 1,8 \\ 0 & 0 & 3,0 & 1,5 & 1,1 & 0 & 1,8 \\ 0,3 & 0 & 1,5 & 0 & 1,8 & 0 & 3,0 \\ 0 & 0 & 7,5 & 0 & 1,8 & 0 & 3,0 \\ 0 & 4,3 & 0 & 0 & 1,1 & 2,7 & 0 \\ 0,6 & 0 & 0 & 0 & 0,7 & 0,9 & 0,6 \\ 0,9 & 0 & 0 & 0,7 & 0 & 1,2 \\ 0 & 0 & 0 & 0,4 & 0 & 0,6\end{array}$


TABLA 2 (continuación)

\begin{tabular}{|c|c|c|c|c|c|c|c|}
\hline Ítem trófico & RS & $\mathrm{CC}$ & $\mathrm{CA}$ & LA & REG & EN-P & EN-A \\
\hline \multicolumn{8}{|l|}{ Chlorophyta } \\
\hline Ulva rigida C. Agardh & 59,2 & 41,4 & 26,9 & 56,9 & 46,4 & 45,5 & 47,0 \\
\hline Pseudoulvella sp. & 34,2 & 32,9 & 29,9 & 52,3 & 37,1 & 18,8 & 49,4 \\
\hline Codium dimorphum Svedelius & 30,3 & 18,6 & 31,3 & 33,8 & 28,4 & 0 & 47,6 \\
\hline Enteromorpha sp. & 25,0 & 20,0 & 14,9 & 15,4 & 19,1 & 17,0 & 20,5 \\
\hline Blidingia minima (Kützing) Kylin & 18,4 & 2,9 & 1,5 & 27,7 & 12,6 & 8,0 & 15,7 \\
\hline Chaetomorpha sp. & 7,9 & 1,4 & 16,4 & 16,9 & 10,4 & 8,9 & 11,4 \\
\hline Cladophora fascicularis (Mertens) Kützing & 14,5 & 4,3 & 13,4 & 7,7 & 10,1 & 0,9 & 16,3 \\
\hline Ulothrix flacca (Dillwyn) Thuret & 13,2 & 0 & 7,5 & 7,7 & 7,2 & 0 & 12,0 \\
\hline Rhizoclonium sp. & 7,9 & 5,7 & 4,5 & 7,7 & 6,5 & 3,6 & 8,4 \\
\hline Chaetomorpha firma Levring & 5,3 & 0 & 0 & 13,8 & 4,7 & 0 & 7,8 \\
\hline Urospora sp. & 7,9 & 1,4 & 3,0 & 6,2 & 4,7 & 0,9 & 7,2 \\
\hline Ulvella lens Crouan & 10,5 & 2,9 & 0 & 0 & 3,6 & 1,8 & 4,8 \\
\hline Rama novazelandiae (J. Agardh) Chapman & 0 & 0 & 9,0 & 3,1 & 2,9 & 0 & 4,8 \\
\hline Pseudopringsheimia sp. & 5,3 & 0 & 0 & 0 & 1,4 & 0 & 2,4 \\
\hline Enteromorpha compressa (Linnaeus) Greville & 2,6 & 0 & 0 & 0 & 0,7 & 0,9 & 0,6 \\
\hline Cladophoropsis herpestica (Montagne) Howe & 0 & 0 & 1,5 & 0 & 0,4 & 0,9 & 0 \\
\hline
\end{tabular}

Phaeophyta

Ralfsia sp.

Ectocarpus acutus Setchell \& Gardner

Lessonia nigrescens Bory

Macrocystis integrifolia Bory

Petalonia fascia (Müller) Kuntze

Colpomenia spp.

Sphacelaria furcigera Küntzing

Endarachne binghamiae J. Agardh

Glossophora kunthii (C. Agardh) J. Agardh

Adenocystis utricularis (Bory) Skottsberg

Pilayella littoralis (Linnaeus) Kjellman

Myriogloia chilensis (Montagne) Llaña

$\begin{array}{ccccccc}26,3 & 21,4 & 26,9 & 35,4 & 27,3 & 6,3 & 41,6 \\ 14,5 & 10,0 & 34,3 & 32,3 & 22,3 & 6,3 & 33,1 \\ 1,3 & 0 & 9,0 & 7,7 & 4,3 & 0 & 7,2 \\ 5,3 & 0 & 0 & 10,8 & 4,0 & 2,7 & 4,8 \\ 2,6 & 7,1 & 1,5 & 1,5 & 3,2 & 0 & 5,4 \\ 6,6 & 2,9 & 1,5 & 0 & 2,9 & 4,5 & 1,8 \\ 0 & 0 & 9,0 & 0 & 2,2 & 0 & 3,6 \\ 0 & 0 & 0 & 6,2 & 1,4 & 0 & 2,4 \\ 2,6 & 2,9 & 0 & 0 & 1,4 & 0 & 2,4 \\ 2,6 & 0 & 0 & 1,5 & 1,1 & 1,8 & 0,6 \\ 2,6 & 0 & 0 & 0 & 0,7 & 1,8 & 0 \\ 0 & 1,4 & 0 & 0 & 0,4 & 0,9 & 0\end{array}$

Otras algas

Bacillarophyceae

$\begin{array}{ccccccc}52,6 & 50,0 & 58,2 & 53,8 & 53,6 & 4,5 & 86,7 \\ 9,2 & 17,1 & 7,5 & 21,5 & 13,7 & 0,9 & 22,3 \\ 9,2 & 4,3 & 3,0 & 20,0 & 9,0 & 0 & 15,1 \\ 1,3 & 4,3 & 3,0 & 6,2 & 3,6 & 0 & 6,0\end{array}$

Cyanophyta nd 
TABLA 2 (continuación)

\begin{tabular}{|c|c|c|c|c|c|c|c|}
\hline Ítem trófico & RS & $\mathrm{CC}$ & $\mathrm{CA}$ & LA & REG & EN-P & EN-A \\
\hline \multicolumn{8}{|l|}{ Invertebrados } \\
\hline Cirripedia nd & 48,7 & 40,0 & 37,3 & 38,5 & 41,4 & 17,9 & 57,2 \\
\hline Notochthamalus scabrosus (Darwin) & 38,2 & 1,4 & 0 & 36,9 & 19,4 & 19,6 & 19,3 \\
\hline Bryozoa nd & 21,1 & 1,4 & 17,9 & 15,4 & 14,0 & 0 & 23,5 \\
\hline Demospongiae nd & 9,2 & 8,6 & 10,4 & 27,7 & 13,7 & 0 & 22,9 \\
\hline Jehlius cirratus (Darwin) & 26,3 & 7,1 & 0 & 3,1 & 9,7 & 15,2 & 6,0 \\
\hline Perumytilus purpuratus (Lamarck) & 23,7 & 1,4 & 0 & 0 & 6,8 & 8,0 & 6,0 \\
\hline Amphipoda nd & 7,9 & 1,4 & 4,5 & 10,8 & 6,1 & 0 & 10,2 \\
\hline Nematoda nd & 2,6 & 4,3 & 4,5 & 12,3 & 5,8 & 0 & 9,6 \\
\hline Foraminifera nd & 2,6 & 1,4 & 13,4 & 4,6 & 5,4 & 0,9 & 8,4 \\
\hline Ostracoda nd & 5,3 & 0 & 1,5 & 15,4 & 5,4 & 0 & 9,0 \\
\hline Semimytilus algosus (Gould) & 6,6 & 2,9 & 6,0 & 6,2 & 5,4 & 1,8 & 7,8 \\
\hline Balanus flosculus Darwin & 10,5 & 1,4 & 0 & 6,2 & 4,7 & 7,1 & 3,0 \\
\hline Hydrozoa nd & 2,6 & 2,9 & 0 & 13,8 & 4,7 & 0 & 7,8 \\
\hline Austrolittorina araucana Orbigny & 5,3 & 1,4 & 7,5 & 3,1 & 4,3 & 2,7 & 5,4 \\
\hline Hyale sp. & 6,6 & 2,9 & 1,5 & 1,5 & 3,2 & 3,6 & 3,0 \\
\hline Isopoda nd & 1,3 & 2,9 & 6,0 & 0 & 2,5 & 0,9 & 3,6 \\
\hline Echinolittorina peruviana (Lamarck) & 7,9 & 0 & 1,5 & 0 & 2,5 & 0 & 4,2 \\
\hline Liopetrolisthes mitra (Dana) & 0 & 0 & 3,0 & 4,6 & 1,8 & 0,9 & 2,4 \\
\hline Tardigrada nd & 5,3 & 1,4 & 0 & 0 & 1,8 & 0,9 & 2,4 \\
\hline Acari nd & 1,3 & 1,4 & 1,5 & 1,5 & 1,4 & 0 & 2,4 \\
\hline Polychaeta nd & 2,6 & 0 & 1,5 & 1,5 & 1,4 & 0,9 & 1,8 \\
\hline Balanus laevis Darwin & 0 & 0 & 0 & 4,6 & 1,1 & 0 & 1,8 \\
\hline Spirorbidae nd & 0 & 0 & 3,0 & 1,5 & 1,1 & 0,9 & 1,2 \\
\hline Scurria ceciliana (Orbigny) & 2,6 & 0 & 0 & 0 & 0,7 & 0,9 & 0,6 \\
\hline Scurria sp. & 2,6 & 0 & 0 & 0 & 0,7 & 0,9 & 0,6 \\
\hline Tegula atra (Lesson) & 2,6 & 0 & 0 & 0 & 0,7 & 1,8 & 0 \\
\hline Diloma nigerrima (Gmelin) & 0 & 0 & 1,5 & 0 & 0,4 & 0 & 0,6 \\
\hline Lottia orbigny (Dall) & 0 & 0 & 1,5 & 0 & 0,4 & 0,9 & 0 \\
\hline Nemertea nd & 1,3 & 0 & 0 & 0 & 0,4 & 0 & 0,6 \\
\hline Scurria plana (Philippi) & 0 & 1,4 & 0 & 0 & 0,4 & 0 & 0,6 \\
\hline Scurria variabilis (Sowerby) & 0 & 0 & 1,5 & 0 & 0,4 & 0 & 0,6 \\
\hline Tetrapygus niger Molina & 1,3 & 0 & 0 & 0 & 0,4 & 0,9 & 0 \\
\hline
\end{tabular}

Larvas de invertebrados

Nauplius

Diptera

Cypris

Veliger

Megalopa

Zoea

$\begin{array}{ccccccc}2,6 & 0 & 1,5 & 1,5 & 1,4 & 0 & 2,4 \\ 1,3 & 0 & 0 & 1,5 & 0,7 & 0,9 & 0,6 \\ 0 & 0 & 0 & 1,5 & 0,4 & 0,9 & 0 \\ 0 & 0 & 1,5 & 0 & 0,4 & 0 & 0,6 \\ 0 & 0 & 0 & 1,5 & 0,4 & 0 & 0,6 \\ 0 & 0 & 1,5 & 0 & 0,4 & 0 & 0,6\end{array}$


La amplitud de nicho trófico de E. niger a nivel regional fue muy alta $(28,0$ integrando todos los datos obtenidos), al igual que los valores globales a nivel comunitario (integrando datos estacionales) en los cuales no se observó una tendencia latitudinal (Tabla 1). El promedio estacional de amplitud de nicho en cada comunidad (menor que los valores globales por un efecto de tamaño de muestra) mostró una baja variabilidad (Tabla 1) y fue menor en Caleta Constitución y Caleta Angosta que en Río Seco y Lagunillas, aunque estas diferencias no fueron significativas $\left(\mathrm{F}_{3,28}=\right.$ $0,61 ; \mathrm{P}=0,611)$.

La riqueza dietaria promedio de E. niger en cada comunidad y estación fluctuó entre 1,33 \pm 0,21 (EE) y 16,22 $\pm 1,44$ ítemes por individuo, y su variación espacio-temporal mostró una tendencia similar a la amplitud de nicho con valores más bajos en Caleta Constitución y Caleta Angosta que en Río Seco y Lagunillas (Fig. 3). No hubo diferencias significativas entre comunidades $\left(\mathrm{F}_{3,246}=1,42 ; \mathrm{P}=0,263\right)$, pero sí entre estaciones $\left(\mathrm{F}_{7,246}=8,28 ; \mathrm{P}<\right.$
0,00001), con una interacción significativa entre ambos factores $\left(\mathrm{F}_{21,246}=10,66 ; \mathrm{P}<\right.$ $0,00001)$. Sin embargo la interacción se debería solo a una variación temporal diferencial entre las comunidades sin involucrar alguna tendencia particular de covariación entre los factores. La tendencia más consistente entre las comunidades (Fig. 3) fue el contraste entre los valores de riqueza dietaria entre invierno 2004 y otoño 2005, comparativamente menores pero con un aumento progresivo, y los valores posteriores que muestran un leve descenso hacia invierno 2005 y un aumento secundario hacia el final del período de muestreo. Esta tendencia parece combinar el cambio relativo en riqueza dietaria desde 2004 a 2006 con un efecto estacional asociado al aumento en riqueza en el período de verano-otoño. Lo anterior sugiere un posible efecto depresor del evento El Niño 2004-05 sobre la riqueza dietaria de E. niger, aun cuando esta variable no tuvo una correlación significativa con la TSM a través del período completo de estudio $(\mathrm{r}=-0,039 ; \mathrm{n}=32 ; \mathrm{P}=0,828)$.

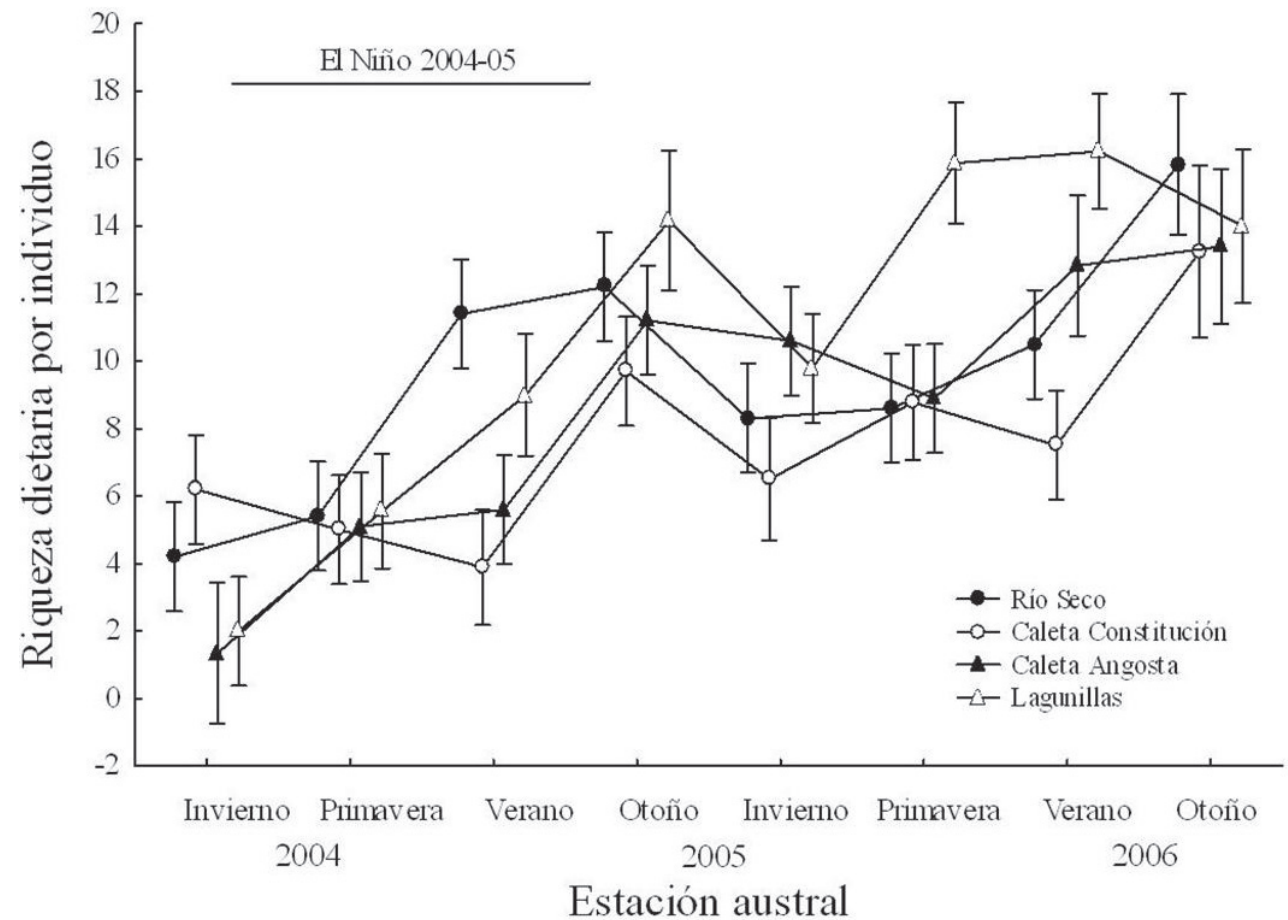

Fig. 3: Variación estacional de la riqueza dietaria por individuo de Enoplochiton niger en las comunidades de estudio. La línea horizontal indica la extensión temporal del evento El Niño 20042005. Los valores corresponden a medias \pm intervalo de confianza del $95 \%$.

Seasonal variation of dietary richness per individual of Enoplochiton niger in the study communities. The horizontal line inside the graph marks the temporal extent of the 2004-2005 El Niño event. Values are means $\pm 95 \%$ confidence intervals. 
Composición taxonómica de la dieta

La composición taxonómica de la dieta de $E$. niger no mostró variaciones entre comunidades (nMDS: estrés $=0,16$; ANOSIM: $\mathrm{R}$ global $=$ $0,042 ; \mathrm{P}=0,18)$, pero sí entre los períodos de presencia y ausencia de El Niño 2004-2005 (R global $=0,634 ; \mathrm{P}=0,01 ;$ Fig. 4). Esta diferencia se debió a dos aspectos distintos. Por una parte, 10 de los ítemes consumidos por $E$. niger durante el período de El Niño (cuatro invertebrados: Tegula atra, Tetrapygus niger, Lottia orbigny, larvas Cypris; seis algas: Ceramium rubrum, Chondria californica, Rhodymenia sp., Cladophoropsis herpestica, Myriogloia sp., Pilayella littoralis) ya no estuvieron presentes en la dieta durante el período posterior. Por otra parte, el número total de ítemes consumidos aumentó desde 61 en el período El Niño hasta 89 en el período posterior, lo cual se evidenció a su vez en cada grupo taxonómico mayor (algas: Rodhophyta de 18 a 25, Chlorophyta de 11 a 15, Phaeophyta de siete a 10; invertebrados: de 23 a 35). La ocurrencia temporal en la dieta (proporción de estaciones de muestreo) de cada uno de los ítemes consumidos por E. niger durante los períodos de presencia y ausencia del evento El Niño 2004-2005 se muestra en la Tabla 2.

\section{DISCUSIÓN}

Enoplochiton niger es claramente un consumidor generalista y polífago, aunque principalmente herbívoro con un consumo vegetal donde destacan las algas incrustantes, tanto calcificadas y no calcificadas, y algas de crecimiento rastrero o en cojín. Algo similar ocurre con su consumo animal, centrado en invertebrados sésiles que cubren la superficie del substrato rocoso. Estas características, sumadas a su gran tamaño (con un máximo de ca. $20 \mathrm{~cm}$ en los individuos muestreados), sugieren que esta especie puede tener un alto impacto sobre los patrones de ocupación de

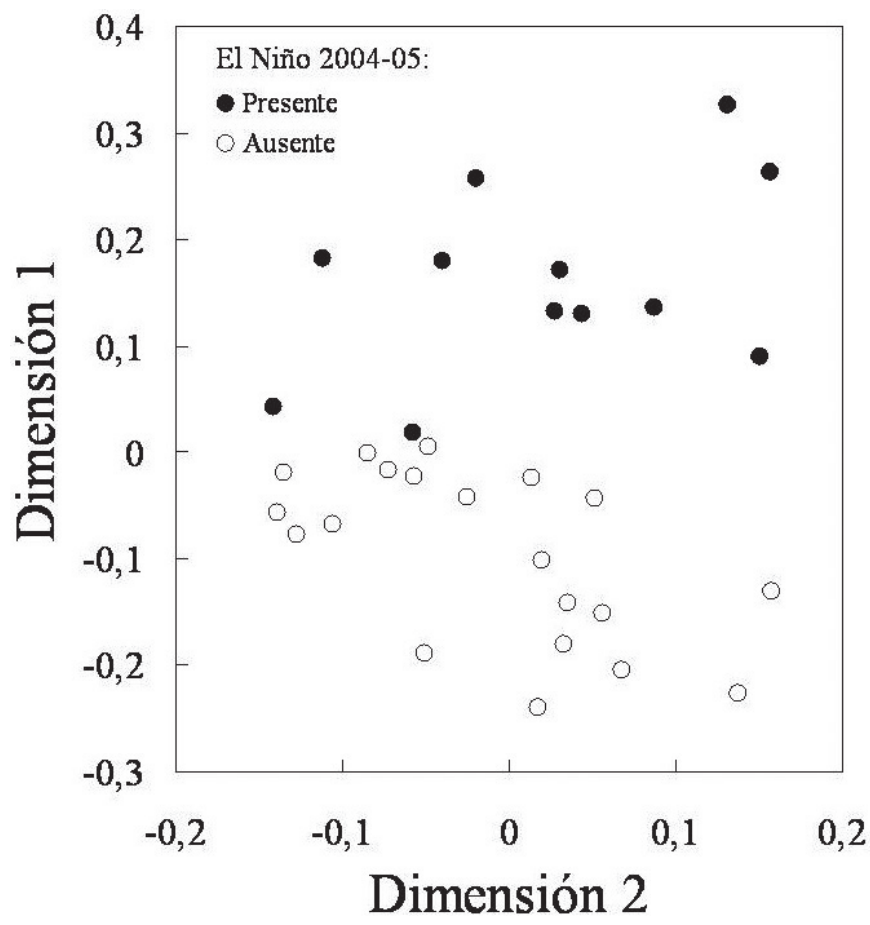

Fig. 4: Análisis de ordenación (nMDS) de la composición taxonómica de la dieta de Enoplochiton niger, registrada para 32 combinaciones muestrales de comunidad*estación clasificadas de acuerdo a la presencia (puntos negros) o ausencia (puntos blancos) del evento El Niño 2004-2005.

Ordination analysis (nMDS) of the taxonomic composition of the diet of Enoplochiton niger, recorded for 32 combinations of community*season classified according with the presence (filled dots) or absence (open dots) of the 2004-2005 El Niño event. 
espacio en la comunidad intermareal, particularmente debido a su acción de pastoreo tipo "bulldozer". Nuestros resultados confirman la alta capacidad de E. niger para controlar el asentamiento y abundancia de otros organismos especialmente en estados tempranos (Camus 1994), sugiriendo que puede ser uno de los agentes bióticos con mayor efecto en los patrones de riqueza y composición de especies en el intermareal rocoso del norte de Chile. Debe notarse que nuestra estimación de la densidad de E. niger en la Tabla 1 incluyó las unidades muestrales con valor cero, particularmente de la zona intermareal media donde la especie es poco frecuente, por lo cual los valores son bajos. No obstante, E. niger puede alcanzar altas densidades en los lugares específicos del hábitat en que está presente, y en este sentido, planteamos que E. niger es uno de los pastoreadores de mayor importancia ecológica en las costas rocosas del norte de Chile, pero lamentablemente uno de los menos conocidos, ya que en apariencia tanto en Chile como en Perú no existe ningún estudio poblacional o comunitario sobre esta especie además del citado anteriormente (Camus 1994), el cual la incorpora solo de forma indirecta.

Otro aspecto relevante de E. niger es la incorporación de $\sim 40 \%$ de ítemes animales en su dieta, lo cual, al igual que las demás especies de chitones del norte de Chile, lo sindica como un potencial omnívoro (Camus et al. 2008). Los cirripedios fueron uno de los principales ítemes consumidos por E. niger, tanto en forma adulta como larval, similar a lo observado en otras especies como Chiton granosus (Aguilera 2005, Camus et al. 2008). Indicios recientes han evidenciado actividad proteolítica y asimilación efectiva en $C$. granosus alimentado con una dieta animal (Cid $2007^{1}$ ), abriendo la posibilidad de que una fracción no menor del consumo animal por estos herbívoros intermareales constituya un aporte nutricional real. Este fenómeno es bastante probable al menos como parte de una estrategia oportunista, como se ha documentado en chitones en otras regiones (e.g., Latyshev et al. 2004), y tiene numerosas implicancias en cuanto a la restringida visión actual de los consumidores herbívoros. Si estos actúan como omnívoros, entonces integrarían cadenas tróficas más largas, y dado que comparten muchos recursos con varias especies herbívoras, generarían un alto número de loops omnívoros cerrados (véase Polis et al. 1989), participando además como depredadores intragremio debido a su consumo de otros herbívoros que son competidores potenciales (Camus et al. 2008).

Por otra parte, la disminución latitudinal hacia el sur en la densidad de E. niger, una tendencia gradual pero bien definida, es coherente con el acercamiento progresivo a su límite sur de distribución $\left(\sim 30^{\circ} \mathrm{S}\right.$, Valdovinos 1999), el cual según algunos autores se habría contraído desde una posición más al sur $\left(\sim 33^{\circ}\right.$ S) en los últimos 55 años por efecto de cambios ambientales (Rivadeneira \& Fernández 2005). Es posible que estos últimos factores puedan tener relación con las diferencias en el patrón de variación temporal en biomasa corporal observadas entre las dos localidades del extremo norte (de tipo estacional) con aquellas dos más al sur (sin tendencia aparente). Por ejemplo, la rigurosidad térmica del ambiente (asociada a los niveles de radiación solar y temperatura del aire, entre otros) varía fuertemente con la latitud y se atenúan hacia el sur (e.g., Muñoz et al. 2005, Thiel et al. 2007, Muñoz et al. 2008), al igual que las tasas de calentamiento de la TSM en las últimas décadas (Rivadeneira \& Fernández 2005). De hecho la riqueza y abundancia de herbívoros intermareales en el extremo norte puede mostrar fuertes variaciones estacionales, aumentando hacia otoño-invierno que serían las épocas más benignas (Camus 1994). No obstante, esta variación temporal diferencial en biomasa corporal con la latitud requiere investigación adicional.

A diferencia de la biomasa corporal, la variación en riqueza dietaria de E. niger no fue estadísticamente distinta entre las localidades estudiadas, mostrando cambios temporales importantes pero con un patrón relativamente similar. Esto sugiere que el patrón temporal común podría estar vinculado en parte a determinantes regionales sobreimpuestas a las variaciones locales. En este sentido, el evento El Niño 2004-2005 aparece como una primera explicación potencial de los cambios observados. Un fenómeno similar se registró en los patrones de riqueza dietaria del erizo negro Tetrapygus niger en las mismas localidades y tiempos (Navarrete et al. 2008), aunque con efectos más contrastantes entre los períodos de 
presencia y ausencia del evento El Niño. De hecho la riqueza dietaria de $T$. niger mostró una clara asociación negativa con la TSM, lo cual no ocurrió con E. niger. No obstante, en ambos casos se registraron variaciones dietarias entre los dos períodos (principalmente un cambio en el número de ítemes dietarios), que aun cuando pudieran estar asociadas a la ocurrencia del evento El Niño no necesariamente se deberían a una limitación de recursos. La Tabla 2 muestra que si bien varias especies de algas e invertebrados fueron mucho menos frecuentes en la dieta de E. niger durante El Niño, otras que son comunes en la zona intermareal fueron consumidas siempre en alta proporción. Esto sugiere que un posible efecto de El Niño sobre la alimentación de E. niger podría implicar eventuales variaciones en la disponibilidad de recursos pero también posibles restricciones fisiológicas. Adicionalmente, es interesante notar que la variación dietaria que podría vincularse a la presencia versus ausencia de El Niño es más bien cuantitativa que cualitativa, pese a que la composición taxonómica de las comunidades estudiadas varía cerca de 40-50\% entre estaciones consecutivas (Camus 2008). Este alto nivel de recambio comunitario supondría a su vez cambios importantes en la composición dietaria de E. niger, lo que sin embargo no ocurre.

Por último, nuestros resultados no mostraron ninguna asociación clara entre las variaciones de mesoescala en intensidad de surgencia y los patrones dietarios o el tamaño corporal de E. niger, como podría haberse esperado de acuerdo a resultados obtenidos en Chile central por otros autores (e.g., Nielsen \& Navarrete 2004, Wieters 2005). Aun cuando el evento El Niño pudiera haber afectado el ingreso de nutrientes o el régimen térmico en las localidades asociadas a una mayor frecuencia e intensidad de surgencia (Caleta Constitución y Lagunillas; Vásquez et al. 1998, Camus \& Andrade 1999, Thiel et al. 2007), las tendencias no cambiaron en el período posterior a El Niño. Por ejemplo, si bien la riqueza dietaria en las últimas estaciones de estudio fue alta en Lagunillas, fue baja en Caleta Constitución, donde a su vez fue menor que en Caleta Angosta y Río Seco (ambos con baja surgencia). Por otra parte, el régimen térmico local de TSM en distintos puntos del norte de Chile puede ser bastante diferente al esperado por la variación estacional o interanual, como ocurre en el área de Antofagasta donde se ubica Caleta Constitución (véase discusión y análisis de anomalías térmicas locales en Camus 2008). No obstante, la falta de información sobre especies consumidoras en el norte de Chile con la importancia ecológica de E. niger no permite aún elaborar hipótesis precisas sobre su comportamiento trófico, y es deseable que esta situación se modifique a corto plazo.

\section{AGRADECIMIENTOS}

Agradecemos el financiamiento otorgado por CONICYT (proyecto FONDECYT $\mathrm{N}^{\circ}$ 1040425), y la asistencia en terreno y laboratorio por Daniela López, Karen Daroch, Romina Seguel y Christian Ibáñez.

\section{LITERATURA CITADA}

AGUiLERA M (2005) Cirripedios en la dieta del molusco herbívoro Chiton gransosus Frembly 1827 (Mollusca: Placophora) presente en el intermareal rocoso de Chile. Investigaciones Marinas (Chile) 33: 109-113.

BOYLE PR (1977) The physiology and behaviour of chitons (Mollusca: Polyplacophora). Oceanography and Marine Biology: an Annual Review 15: 461-509.

CAMUS PA (1994) Recruitment of the intertidal kelp Lessonia nigrescens Bory in northern Chile: successional constraints and opportunities. Journal of Experimental Marine Biology and Ecology 184: 171-181.

CAMUS PA (2008) Understanding biological impacts of ENSO on the eastern Pacific: an evolving scenario. International Journal of Environment and Health 2: 5-19.

CAMUS PA \& YN ANDRADE (1999) Diversidad de comunidades intermareales rocosas del norte de Chile: el supuesto del enriquecimiento por efecto de la surgencia costera. Revista Chilena de Historia Natural 72: 389-410.

CAMUS PA, K DAROCH \& LF OPAZO (2008) Potential for omnivory and apparent intraguild predation in rocky intertidal herbivore assemblages from northern Chile. Marine Ecology Progress Series 361: $35-45$

CLARKE KR (1993) Non-parametric multivariate analyses of changes in community structure. Australian Journal of Ecology 18: 117-143.

CPC (2007) Cold \& warm episodes by season. Climate Prediction Center, NOAA, USA. w w w . c p c.nce p.noa a.gov/product s/ analysis_monitoring/ensostuff/ensoyears.shtml.

HAWKINS SJ \& RG HARTNOLL (1983) Grazing of the intertidal algae by marine invertebrates Oceanography and Marine Biology: an Annual Review 21: 195-282. 
KELAHER BP \& VJ COLE (2005) Variation in abundance and size-structure of populations of the small chiton Acanthochitona retrojecta. Journal of Molluscan Studies 71: 154-151.

LANGER PD (1983) Diet analysis for three subtidal coexisting chitons from the Northwestern Atlantic (Mollusca: Polyplacophora). The Veliger 25: 370372.

LATYSHEV NA, AS KHARDIN, SP KASYANOV \& MB IVANOVA (2004) A study in the feeding ecology of chitons using analysis of gut contents and fatty acid markers. Journal of Molluscan Studies 70: 225230.

LEVINS R (1968) Evolution in changing environments: some theoretical explorations. Princeton University Press, Princeton, New Jersey, USA. 120 pp.

LUBCHENCO J \& SD GAINES (1981) A unified approach to marine plant-herbivore interactions. I. Populations and communities. Annual Review of Ecology and Systematics 12: 405-437.

MARKEL RW \& RE DE WREEDE (1998) Mechanisms underlying the effect of the chiton Katharina tunicata on the kelp Hedophyllum sessile: Size escapes and indirect effects. Marine Ecology Progress Series 166:151-161

MUÑOZ JLP, GR FINKE, PA CAMUS \& F BOZINOVIC (2005). Thermoregulatory behavior, heat gain and thermal tolerance in the periwinkle Echinolittorina peruviana in central Chile. Comparative Biochemistry and Physiology A 142: 92-98.

MUÑOZ JLP, PA CAMUS, FA LABRA, GR FINKE \& F BOZINOVIC (2008) Thermal constraints on daily patterns of aggregation and density along an intertidal gradient in the periwinkle Echinolittorina peruviana. Journal of Thermal Biology 33: 149156.

NAVARRETE AH, PA CAMUS \& LF OPAZO (2008) Variación ambiental y patrones dietarios del erizo negro Tetrapygus niger en costas intermareales rocosas del norte de Chile. Revista Chilena de Historia Natural 81: 305-319.

NIELSEN KJ \& SA NAVARRETE (2004) Mesoscale regulation comes from the bottom- up: Intertidal interactions between consumers and upwelling. Ecology Letters 7: 31- 41.

OTAÍZA RD \& B SANTELICES (1985) Vertical distribution of chitons (Mollusca: Polyplacophora) in the rocky intertidal zone of central Chile. Journal of Experimental Marine Biology and Ecology 86: 229-240.

OTAIZA RD (1986) Patrones de distribución vertical de chitones y efecto de pastoreo de Chiton granosus en roqueríos intermareales de Chile central. En: Santelices B (ed) Usos y funciones ecológicas de las algas marinas bentónicas: 173-190. Monografías Biológicas 4. Ediciones Universidad Católica de Chile, Santiago, Chile.

OTWAY NM (1994) Population ecology of the low-shore chitons Onithochiton querinus and Plaxiphora albida. Marine Biology 121: 105-116.

PIMM SL \& JH LAWTON (1978) On feeding on more than one trophic level. Nature 275: 542-544.
POLIS GA, CA MYERS \& RD HOLT (1989) The ecology and evolution of intraguild predation: Potential competitors that eat each other. Annual Review of Ecology and Systematics 20: 297-330.

RIVADENEIRA MM \& M FERNÁNDEZ (2005) Shifts in southern endpoints of distribution in rocky intertidal species along the south-eastern Pacific coast. Journal of Biogeography 32: 203-209.

SANTELICES B, J VÁSQUEZ \& I MENESES (1986) Patrones de distribución y dietas de un gremio de moluscos herbívoros en hábitats intermareales expuestos de Chile central. En: Santelices B (ed) Usos y funciones ecológicas de las algas marinas bentónicas: 147-175. Monografías Biológicas 4. Ediciones Universidad Católica de Chile, Santiago, Chile.

SIEGEL S \& NJ CASTELLAN (1988) Nonparametric statistics for the behavioral sciences. Second edition, McGraw-Hill, London, United Kingdom. $399 \mathrm{pp}$.

SMITH KA \& NM OTWAY (1997) Spatial and temporal patterns of the abundance and effects of disturbance on under-boulder chitons. Molluscan Research 18: 43-57.

STENECK RS \& L WATLING (1982) Feeding capabilities and limitation of herbivorous molluscs: A functional group approach. Marine Biology 68: 299-319.

THIEL M, E MACAYA, E ACUÑA, W ARNTZ, H BASTÍAS, K BROKORDT, PA CAMUS, JC CASTILLA, LR CASTRO, M CORTÉS, CP DUMONT, R ESCRIBANO, M FERNÁNDEZ, DA LANCELLOTTI, JA GAJARDO, CF GAYMER, I GOMEZ, AE GONZÁLEZ, HE GONZÁLEZ, PA HAYE, JE ILLANES, JL IRIARTE, G LUNAJORQUERA, C LUXORO, PH MANRÍQUEZ, V MARÍN, P MUÑOZ, SA NAVARRETE, E PÉREZ, E POULIN, J SELLANES, A SEPÚLVEDA, W STOTZ, F TALA, A THOMAS, CA VARGAS, JA VÁSQUEZ \& A VEGA (2007) The Humboldt Current System of northern and central Chile: oceanographic processes, ecological interactions and socioeconomic feedback. Oceanography and Marine Biology: an Annual Review 45: 195-345.

VALDOVINOS C (1999) Biodiversidad de moluscos chilenos: base de datos taxonómica y distribucional. Gayana Zoología (Chile) 63: 111-164.

VÁSQUEZ JA, PA CAMUS \& FP OJEDA (1998) Diversidad, estructura y funcionamiento de ecosistemas litorales rocosos del norte de Chile. Revista Chilena de Historia Natural 71: 479-499.

VEGA JM, JA VÁSQUEZ \& AH BUSCHMANN (2005) Biología poblacional de huirales submareales de Macrocystis integrifolia y Lessonia trabeculata (Laminariales, Phaeophyceae) en un ecosistema de surgencia del norte de Chile: variabilidad interanual y El Niño 1997-1998. Revista Chilena de Historia Natural 78: 33-50

WIETERS EA (2005) Upwelling control of positive interactions over mesoscales: a new link between bottom-up and top-down processes on rocky shores. Marine Ecology Progress Series 301: 43-54. 